\title{
Autoextraction of superficial corneal foreign bodies
}

\author{
T. P. AGRAWAL
}

Department of Ophthalmology, Goa Medical College, Panaji-Goa, India.

It is not uncommon for foreign bodies such as dust and coal particles to enter the eye when no medical facilities are available. Because of its exposed position, the cornea is more vulnerable than the conjunctiva and over three-quarters of retained surface foreign bodies lodge on the cornea (Roper-Hall, i964).

Conjunctival foreign bodies on the bulbar conjunctiva or in the lower fornix or the sulcus subtarsalis present little difficulty, but the cornea is too sensitive to be touched without a local anaesthetic, and the eye should not be rubbed to relieve irritation. By the following simple method the patient may be able to remove a foreign body himself.

\section{Method}

The eye should be gently closed. The centre of the skin of the upper lid should be held above the lid margin between the thumb and index finger (Figs $\mathrm{I}$ and 2), jerked abruptly away from the eye (Fig. 3), and then released. The separation of the lid from the eyeball makes a slight noise like smacking the lips. This process may be repeated three or four times. The patient should move the upper lid to feel for the foreign body sensation, the absence of which will indicate that the gritty particle has been successfully dislodged from the cornea. The eye may afterwards be irrigated with clean tap water.

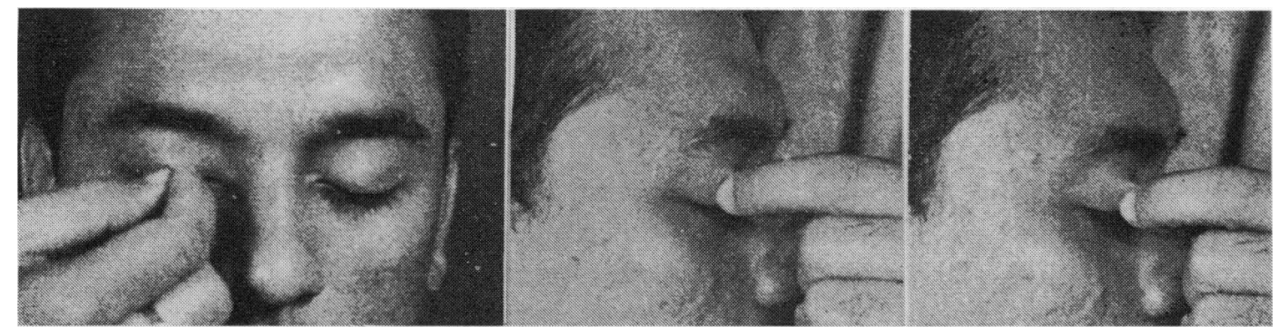

FIG. I Front view, showing skin of FIG. 2 Side view of Fig. I FIG. 3 Side view, showing upper lid held between thumb and index finger skin of upper lid pulled forwards

\section{Discussion}

When the eye is gently closed the conjunctival space filled with tears forms a closed cavity (Duke-Elder, I96I). As the upper lid skin is pulled away from the cornea, a vacuum is created with a sucking effect which is strong enough to draw a superficial foreign body away from the cornea. 
This method may be used as a routine first aid measure in all cases of corneal foreign bodies, as it will not damage the cornea and does not require a local anaesthetic.

The patient must keep the eye closed in order to create a vacuum, and the skin must be pulled suddenly so that the sucking or smacking sound is produced.

\section{Summary}

A simple technique for the autoextraction of superficial corneal foreign bodies is described. It may be attempted as a first-aid measure for the extraction of all corneal foreign bodies.

\section{References}

DUKe-ELDER, s., and WYBAR, K. c. (196r) "System of Ophthalmology", vol. 2, p. 541. Kimpton, London

ROPER-HALl, M. J. (1964) In "Modern Ophthalmology", ed. A. Sorsby, vol. 3, p. 396-397. Butterworths, London 